\title{
Design of a Machine Vision System Based on FPGA
}

\author{
Ju Hua ${ }^{1, a^{*}}$, Li Shu-lin ${ }^{2, b}$ \\ ${ }^{1}$ School of Applied Sciences, University of Science and Technology Liaoning, Anshan, 114051, \\ China \\ ${ }^{2}$ Engineering Training Center, University of Science and Technology Liaoning, Anshan, 114051, \\ China \\ a642468130@qq.com, b1357280818@qq.com
}

Keywords: machine vision, FPGA, Gige Vision

Abstract. In order to realize the system's fast update, a new kind of machine vision solution is designed. It uses video input port and Gige Vision IP , and connects between the system and the computer, which is realized by FPGA technology. The new design plan contains new Gige Visiong standard and the core of Gige Vision IP, to make the system easier and faster, which is the development direction in the future.

\section{Introduction}

Machine vision is to use the machine to replace the human eye to do measurement and judgment. As an extension of human vision and brain, machine vision is one of the symbols of modern industrial automation[1]. In recent years, with the development of computer technology, multimedia technology, digital image processing and analysis theory, and the rapid development of large-scale integrated circuits, machine vision technology has been widely used in research, and has achieved tremendous economic and social benefits.

The machine vision system is composed of 3 parts: image acquisition, image processing and analysis, output and display[2]. This paper introduce a machine vision system based on FPGA , using Gige Vision camera to obtain the image. The system uses the core of Gige Vision IP, and transport protocol based on TCP/IP to realize data communication between FPGA and PC.

\section{FPGA`s advantages in machine vision}

With the improvement of the resolution and the improvement of the compression algorithm, the system performance and architecture of the machine vision system are improved. The system architecture can be selected including the standard cell ASSP, ASIC, and a variety of programmable solutions, such as digital signal processor (DSP), media processor and FPGA. Each frame has its own advantages and disadvantages, and the final choice depends on the terminal equipment requirements and the availability of the solution. Ideal architecture should have the following characteristics: high performance, flexibility, easy to upgrade, low development costs and with the application of mature and yield increase to lower cost migration. FPGA has advantages in parallel computing, repeatedly using, and can be substantial use, which make it a reasonable choice to achieve ideal machine vision architecture[3].

FPGA is one of the best options for improving the integration and reliability of the system. With the continuous improvement of the VLSI process, a single chip has been able to accommodate a million transistors, which makes the function of the FPGA chip can achieve more and more strong, but also to achieve system integration.

In the design of SoC, the development of the design process of FPGA device makes it possible to integrate more and more functions into one chip. In order to achieve this goal, there is a very practical SoC design method, known as the nuclear based design, it will be the system function is divided into different core, using IP (Intellectual Property) core to complete the specific design features.[4] 


\section{Design of machine vision system}

\section{Design principle}

The solution is based on the 3E FPGA Spartan core chip with low power consumption and low power consumption. It provides an integrated solution to combine Camera Link, Gige Vision IP, Gigabit Ethernet MAC platform and optional Applets Visual software. Figure 1 shows the design principle.

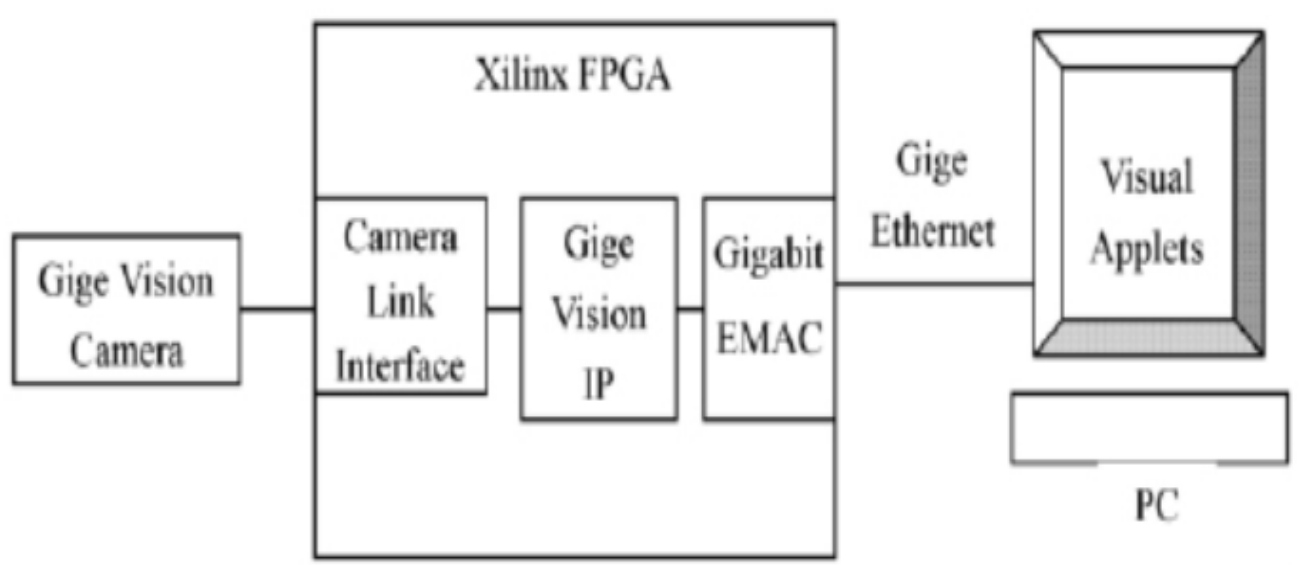

Figure 1. Structure of the machine vision system

\section{Video input module}

The main advantages of Gige Vision camera are the followings:

(1) Progressive scanning;

(2) High clarity, resolution of 4 million pixels;

(3) High frame rate: the highest frame rate reached $200 \mathrm{f} / \mathrm{s}$;

(4) Data without compression, no loss;

(5) Can scan the data for the required output;

(6) The camera parameters can be changed automatically by the software;

(7) Convenient application development.

Besed on the advantages, we choose Gige Vision camera for the system.

\section{Module in FPGA}

FPGA uses Camera Link to perform the interconnection with the camera. Camera Link is a powerful data channel for high-end machine vision. It uses a dedicated cable connection and a standardized communication protocol. Camera Link supports a large amount of data and high data transmission rate, suitable for today's image data transmission requirements.

Gige Vision is a kind of ideal standard for data centralized and distributed processing of single / multi camera and single / multi computer. Standard Gigabit Ethernet is used to allow the use of standard cables and connectors, thereby reducing the cost of material and the integration of the cost and the subsequent growth of the scalability of the Ethernet bandwidth[5].

The Gige Vision IP core used in this program is based on the hardware implementation of Gige Vision data stream protocol version 1. It is composed of Sensor-to-Image GmbH, partner of Xilinx.

The system uses hardware to achieve the data flow channel, which can achieve the maximum throughput of Gigabit ethernet. The structure of the IP kernel is shown in Figure 2. 


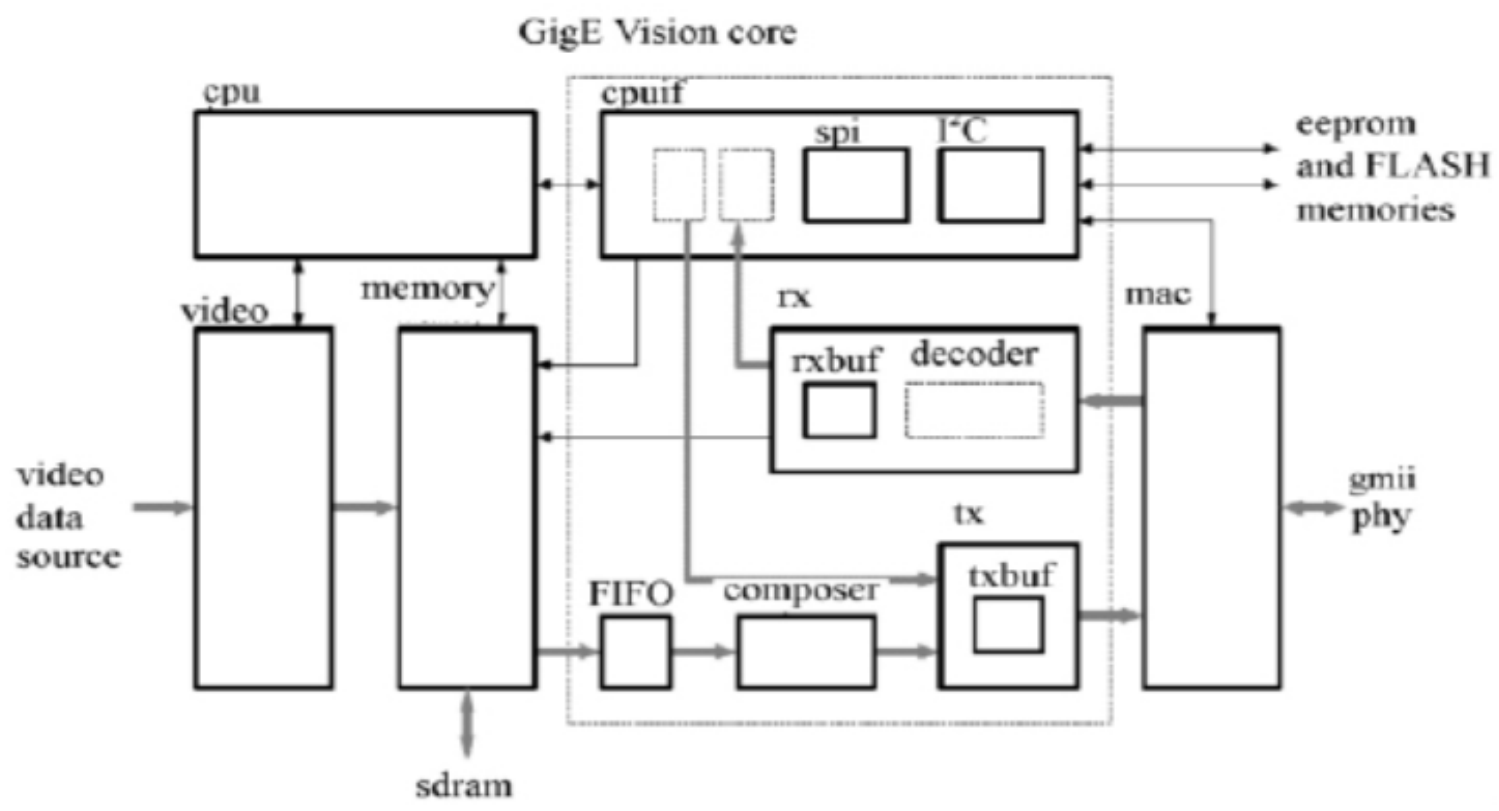

Figure 2. Structure of Gige Vision IP core

The core of Vision Gige is composed of memory controller, Jibit Ethernet MAC, control processor and a video processing unit.

It uses Phantom Trimode MAC IP core, which is developed by Finger Lakes Engineering for Xilinx, to connect FPGA and the computer. Phantom core has unique retiming path, so it can reduce the number of clock buffers and simplify the integration of the core, making it he ideal choice for both the low cost of the Spartan device and the high performance of the Virtex architecture[6].

\section{Host interface design}

The system uses Gigabit Ethernet to connect the computer, and the computer need to install visual applets software.

The Visual Applets, developed by Silicon Software, is the most advanced FPGA design tools. Through graphics interface which is easy to learn and use, it's easy to develop a special image processing configuration FPGA, which is called applet.

The Visual Applets`advantages are:

(1) Make the video card into an efficient image processor;

(2) The potential of modern FPGA can be played by any user;

(3) Image processing solutions can be completed within a few minutes ;

(4) Rapid development of hardware class.

As long as using Applets Visual to link between the various processing modules, the compatibility of various links can be displayed in real time, each module can be linked to different phase relations. After the completion of the design, it takes only a few minutes to produce a compiled file, during which does not no artificial action is needed, the applet's parameter can be immediately setted in the micro-Display, and at the same time, it will automatically generate a visual $\mathrm{C}++\mathrm{SDK}$ project.

\section{Summary}

In this paper, a general machine vision system based on FPGA chip is designed, which can be applied to various occasions such as product line, intelligent vehicle monitoring and management. The 
performance and reliability of the system is greatly improved by using the latest high performance visual standards and the development of the IP core and the Applets Visual software.

\section{References}

[1] Corke P I. Machine Vision[J]. Moldes, 2000, 19(3):0219--6131.

[2] Davies E R. Computer and Machine Vision[M]// Elsevier LTD, Oxford, 2012.

[3] Moradewicz A J, Kazmierkowski M P. Contactless Energy Transfer System With FPGA-Controlled Resonant Converter[J]. IEEE Transactions on Industrial Electronics, 2010, 57(9):3181-3190.

[4] Ling X G, Li Q, Ding Y Y, et al. Design and Implementation of Modular FPGA-Based Multiple-Axis Motion Controller for CNC[J]. Applied Mechanics \& Materials, 2011, 141:233-238.

[5] Isakova N, Basak S, Sonmez A C. FPGA design and implementation of a real-time stereo vision system[C]// Innovations in Intelligent Systems and Applications (INISTA), 2012 International Symposium on. IEEE, 2012:1-5.

[6] Monmasson E, Idkhajine L, Naouar M W. FPGA-based Controllers[J]. Industrial Electronics Magazine IEEE, 2011, 5(1):14-26. 